\title{
Using Meta-Knowledge Mined from Identifiers to Improve Intent Recognition in Conversational Systems
}

\author{
Claudio S. Pinhanez, Paulo Cavalin, Victor Ribeiro, Ana Paula Appel, \\ Heloisa Candello, Julio Nogima, Mauro Pichiliani, Melina Guerra, \\ Maira Gatti de Bayser, Gabriel Malfatti, Henrique Ferreira
}

\author{
IBM Research - Brazil \\ csantosp@br.ibm.com
}

\begin{abstract}
In this paper we explore the improvement of intent recognition in conversational systems by the use of meta-knowledge embedded in intent identifiers. Developers often include such knowledge, structure as taxonomies, in the documentation of chatbots. By using neurosymbolic algorithms to incorporate those taxonomies into embeddings of the output space, we were able to improve accuracy in intent recognition. In datasets with intents and example utterances from 200 professional chatbots, we saw decreases in the equal error rate (EER) in more than $40 \%$ of the chatbots in comparison to the baseline of the same algorithm without the meta-knowledge. The metaknowledge proved also to be effective in detecting out-of-scope utterances, improving the false acceptance rate (FAR) in two thirds of the chatbots, with decreases of 0.05 or more in FAR in almost $40 \%$ of the chatbots. When considering only the well-developed workspaces with a high level use of taxonomies, FAR decreased more than 0.05 in $77 \%$ of them, and more than 0.1 in $39 \%$ of the chatbots.
\end{abstract}

\section{Introduction}

Classification of sentences into a discrete set of classes is a key part of professional conversational systems. In fact, most of those systems require developers to define the different classes, or intents, by enumerating exemplars of each of them, since classification is often performed using $\mathrm{ma}$ chine learning $(M L)$ methods. The process of classifying an input sentence into a specific intent or signaling it as out-of-scope (OOS) of the system is often referred to as intent recognition.

Determining a class solely on a list of exemplars is a practical method to implement ML systems but it is hardly a natural way for human beings to define a class. In real life, people define classes often using a rich mix of symbolic definitions, sometimes taxonomic in nature, such as in "a credit card is a type of bank card", coupled with its sub-classes, for instance, "basic", "premium", and typical features such as "international". People also use exemplars, "card X of bank Y is a credit card", as well as particular examples to describe a sub-class, such as in "card W is an international card". They also use counter examples, either categorically, "a debit card is not a credit card", or in examples, "card $\mathrm{Z}$ is not a credit card". Defining and specifying classes in the real world is, in fact, a cultural, contextual, and linguistic construct, and how people and societies perform this process is a traditional research subject in social sciences, notably in anthropology (Durkheim and Mauss, 1963; Needham, 1979; Bowker and Star, 2000).

This paper explores algorithms for intent recognition which use both the sets of exemplars and taxonomic-like symbolic descriptions of a class to define and train intents in conversational systems using ML methods. We aim not only to provide methods more aligned to everyday class definition practices of developers but also to improve the accuracy of the ML methods. Inspired by a reverse dictionary algorithm (Kartsaklis et al., 2018) and previous work on keyword-based classification (Cavalin et al., 2020), we propose three neuro-symbolic algorithms which combine taxonomic descriptions of classes with traditional exemplar-based supervised learning. We show that those novel algorithms are able to decrease error rates for a significant number of datasets, particularly in the difficult task of detecting OOS cases in real, professional chatbots.

The key idea behind our algorithms is to substitute the typical softmax used in the output layer of a ML text classifier with a space of embeddings of the taxonomic descriptions of the intents. The training process uses the exemplars in standard ways while the recognition process is performed using similarity distances in the embedding output space. 
This is similar to ideas used in zero-shot learning methods (Palatucci et al., 2009; Socher et al., 2013; Akata et al., 2015, 2016), in which classes defined by sub-concepts are also encoded with special embeddings to allow detection of new classes without exemplars.

We tested our algorithms using real datasets by exploring a common practice among developers of conversational systems, who often embed symbolic knowledge as documentation in intent identifiers. In a previous work (Pinhanez et al., 2021), we observed a pattern among developers of using taxonomic-like structures to name the intents in which strings of reoccurring concepts are used to identify and document the different classes. For example, an intent about utterances where users ask for the balance of a credit card may be named "checking_credit_card_balance", while an intent related to finding out the date of payment of the balance could be identified as "asking_credit_card_balance_payment_date".

We call those structures intent proto-taxonomies, and real examples are shown in figures 1 and 2 . In (Pinhanez et al., 2021), we studied the use by developers of intent proto-taxonomies quantitatively and qualitatively, as well as proposed an algorithm to mine this meta-knowledge automatically, and concluded that their use is fairly common in at least one professional chatbot development platform. This paper focuses on the algorithms to use the meta-knowledge and on evaluating their impact on the accuracy of intent recognition.

The paper starts by looking into the recent advances in neuro-symbolic systems and describing briefly the practice of developers of conversational systems of embedding meta-knowledge within the source code of their systems. We follow by describing the proposed three algorithms integrating such meta-knowledge into intent recognition ML algorithms and by evaluating them first with two typical intent recognition datasets, and then with hundreds of workspaces created in a professional tool called here ChatWorks ${ }^{1}$. The results show most of those workspaces can benefit from the techniques described in this paper, notably for OOS detection tasks, often with accuracy improvements of $5 \%$ or more solely derived from the use of the additional symbolic description from the documentation.

\footnotetext{
${ }^{1}$ We use an anonymous name for the tool due to publication restrictions from the platform company.
}

\section{Related Work}

The value and limits of symbolic categorization in AI have been of interest since the early days (Newell, 1973; Richards, 1982; Kosslyn, 2006). But our work fits more in the context of a growing belief that symbolic knowledge needs to be included in ML systems, materialized in the so called neuro-symbolic approaches (Parisotto et al., 2017; Besold et al., 2017; Tenenbaum et al., 2011; Bengio, 2017; Mao et al., 2019; Hudson and Manning, 2019a; De Raedt et al., 2019).

Neuro-symbolic methods "aim to transfer principles and mechanisms between (often nonclassical) logic-based computation and neural computation" (Besold et al., 2017). Such kind of systems are viewed by some researchers as a way to embed high-level knowledge and even some form of "consciousness" into machine learning systems, making the language to develop them closer to "what passes in a man's own mind" (Bengio, 2017).

In recent years, AI has witnessed a myriad of novel neuro-symbolic techniques and their application to different problems, contexts, and scenarios (Parisotto et al., 2017; Manhaeve et al., 2018; d'Avila Garcez et al., 2019; Hudson and Manning, 2019b; De Raedt et al., 2019). For instance, in (Mao et al., 2019), an approach for image understanding is suggested which takes the object-based scene representations and translates sentences into executable, symbolic programs. In (Oltramari et al., 2020), embeddings of knowledge graphs are used as attention layers for tasks such as autonomous driving (AV) and question-answering. And in (Kartsaklis et al., 2018), random walks in a knowledge graph are mapped as sentence embeddings for use in an inverse dictionary problem.

One important requirement for many neurosymbolic systems is to represent knowledge in a structured format such as knowledge graphs, ontologies, or taxonomies (Ji et al., 2020). In some cases, such as the scene ontology for autonomous vehicles in (Oltramari et al., 2020), a lot of effort was needed for manual annotation. Nevertheless, as presented in (Fossati et al., 2015), an unsupervised approach can sometimes be used to mine the meta-knowledge introduced by the experts, such as the classes in Wikipedia pages.

Considering our context, intent identifiers are sometimes described using high-level representations of the class as we detail later. This is similar to what is used in some zero-shot learning 
techniques (Wang et al., 2019) in which classes manually defined by sub-concepts are encoded with special embeddings so new classes can be detected without training (Palatucci et al., 2009; Socher et al., 2013; Akata et al., 2015, 2016; Chen et al., 2016). In (Chen et al., 2016), for example, intent identifiers can be formatted as natural language sentences to learn a model which maps training examples into those sentences, so that the meta-knowledge can be used in zero-shot learning. However, the dataset explored in that work is very limited. Recent work has also demonstrated that intent recognition can be improved by enhancing class representations with "keywords" which are extracted from exemplar utterances considering their most common words (Cavalin et al., 2020).

This work focuses on high level class representations based on taxonomies and aims to explore their usefulness as enhancers of ML intent recognition algorithms. It also explores different ways of embedding taxonomy-like meta-knowledge considering different methods of representation.

\section{The Knowledge Embedded in Intent Identifiers of Conversational Systems}

Most real-world, deployed conversational systems in use today have been built based on the rule-based intent-action paradigm, using platforms such as Luis.ai, Watson Assistant, or Alexa Skills. Each intent corresponds to a desired information or answer from the user and is defined by a set of exemplar utterances by the chatbot developers. During runtime, each utterance from the user is recognized as one of the defined intents or as out-of-scope (OOS), and then the associated action is outputted.

In the context of the chatbots built using the ChatWorks platform explored in this paper, a previous work of the authors of this paper (Pinhanez et al., 2021) has shown that the curators and developers of chatbots often store symbolic knowledge in a taxonomic form about the intent classes in a documentation field called nameId. Figure 1 shows some examples of those nameIds, obtained from a professional finance chatbot, here translated from the original in Portuguese and anonymized to preserve confidential information.

This practice was studied in workshops with developers (Pinhanez et al., 2021), which determined that the goal of the taxonomic description is to provide the intent classes with a summarized description of each intent. Such taxonomic naming

\begin{tabular}{|c|l|}
\hline intent & INTENT ID (translated and anonymized) \\
\hline 1 & inv_XXX-fidelity-concept-special_inv-kareen_27-08-YY \\
\hline 2 & inv_XXX-general-check-balance \\
\hline 3 & inv_XXX-general-check-investment_period \\
\hline 4 & inv_XXX-general-check-issue_time-special_inv \\
\hline 5 & inv_XXX-general-check-rate-management \\
\hline 6 & inv_XXX-general-concept-special_inv \\
\hline 7 & inv_XXX-general-desambiguation-check_withdraw_date-- \\
\hline 8 & inv_XXX-general-desambiguation-modalities-special_inv \\
\hline 9 & inv_XXX-general-desambiguation-withdraw-- \\
\hline 10 & inv_XXX-general-income_tax-bank_website \\
\hline 11 & inv_XXX-general-liquidity-special_inv \\
\hline 12 & inv_XXX-general-withdraw-minimum_time-special_inv \\
\hline 13 & inv_XXX-general-withdraw-using_selfservice-management \\
\hline 14 & inv_XXX-easy-concept-special_inv-kareen_27-08-YY \\
\hline 15 & inv_XXX-prefix-concept-special_inv-kareen_27-08-YY \\
\hline 16 & inv_XXX-prefix-investment-minimum_time-bank_website \\
\hline 17 & inv_XXX-prefix-withdraw-procedure-bank_website \\
\hline 18 & inv_XXX-withdraw-phone_app-- \\
\hline 19 & inv_XXX-withdraw-using_app_bank-management \\
\hline 20 & inv_XXX-withdraw-using_internet_banking-- \\
\hline
\end{tabular}

Figure 1: Some nameIds of intents of a finance chatbot.

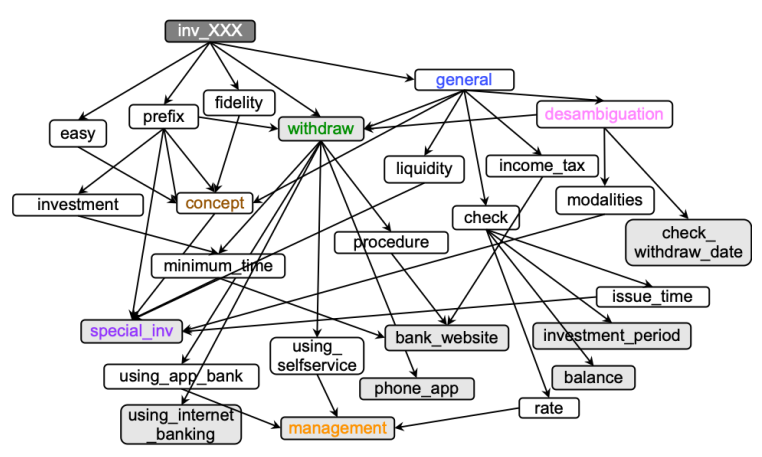

Figure 2: The intent proto-taxonomy associated to the intents of fig. 1.

patterns are also common in the way people organize files and e-mails in computers (Civan et al., 2008; Whittaker et al., 2011) and how software developers name functions (Yang et al., 2019).

As described in (Pinhanez et al., 2021), such knowledge-embedding practices are, in fact, fairly common among curators in the ChatWorks platform. Using the algorithm reproduced in appendix A, taxonomic-like symbolic knowledge was automatically extracted from workspaces defining almost 7,000 professional chatbots, in two different languages. By considering the different words in the nameIds as basic concepts and consecutive concepts as having connections between them, we can structure the set of nameIds as a very basic knowledge graph (Ehrlinger and Wöß, 2016), hereby referred as an intent proto-taxonomy.

Figure 2 depicts the intent proto-taxonomy associated to the nameIds in fig. 1. Next, as proposed 
in (Pinhanez et al., 2021), it is possible to compute the taxonomy rate of a workspace by calculating the ratio between the number of intents with taxonomies and the total number of intents.

In 3,840 professsional workspaces in the English language, it was found that $76 \%$ of them had a taxonomy rate above $10 \%$, almost $52 \%$ had a taxonomy rate above $50 \%$, and $16 \%$ had a very high taxonomy rate, above $90 \%$. Moreover, the distribution followed a sort of "step" function where, as the threshold of 32 in the number of intents in a workspace was crossed, the majority of the workspaces had a taxonomy rate of more than $50 \%$. It seems that, as the complexity of the workspace increases with the number of intents, more often developers resort to document them using an intent proto-taxonomy (see appendix B for details).

The use in our work of the intent prototaxonomies as a symbolic description of classes is feasible because: (1) they are part of the documentation of the conversational system, so there is no need of acquiring knowledge from experts; (2) they are easily mined, as described in appendix A.

\section{Using Taxonomic Intent Descriptions to Improve Intent Recognition}

We present now a formal description of the methodology employed in this work which takes advantage of the intent proto-taxonomies using a neurosymbolic approach. It expands some previous work which focused on the use of keywords as the source of symbolic information (Cavalin et al., 2020). .

\subsection{Embedding the Set of Classes}

An intent classification method is a function $D$ which maps a set of sentences (potentially infinite) $S=\left\{s_{1}, s_{2}, \ldots\right\}$ into a finite set of classes $\Omega=$ $\left\{\omega_{1}, \omega_{2}, \ldots, \omega_{c}\right\}$ :

$$
D: S \rightarrow \Omega \quad D(s)=\omega_{i}
$$

To enable a numeric, easier handling of the input text, an embedding $\xi: S \rightarrow \mathbb{R}^{n}$ is often used, mapping the space of sentences $S$ into a vector space $\mathbb{R}^{n}$, and defining a classification function $E$ : $\mathbb{R}^{n} \rightarrow \Omega$ such that $D(s)=E(\xi(s))$. In most intent classifiers, $E$ is composed of a function $M$ which computes the likelihood of $s$ being in a given class, often a neural network, followed by some sort of argmax function. Typically, softmax + argmax is used, noted simply as softmax here:

$$
S \stackrel{\xi}{\rightarrow} \mathbb{R}^{n} \stackrel{M}{\rightarrow} \mathbb{R}^{c} \stackrel{\text { softmax }}{\rightarrow} \Omega
$$

This paper explores how to use embeddings in the output side of the classification function, that is, by embedding the set $\Omega$ of classes into another vector space $\mathbb{R}^{m}$, in some ways resembling the combination of object-based recognition and symbolic programming in (Mao et al., 2019). Instead, we combine here standard intent recognition methods with an encoding of taxonomies in knowledge graph-like structures. The idea is to use class embedding functions which somehow capture the knowledge in the intent proto-taxonomies.

Formally, we use a class embedding function $\psi: \Omega \rightarrow \mathbb{R}^{m}$, its inverse $\psi^{-1}$, and a function $M: \mathbb{R}^{n} \rightarrow \mathbb{R}^{m}$ to map the two vector spaces so $D(s)=\psi^{-1}(M(\xi(s)))$.

$$
S \stackrel{\xi}{\rightarrow} \mathbb{R}^{n} \stackrel{M}{\rightarrow} \mathbb{R}^{m} \stackrel{\psi^{-1}}{\rightarrow} \Omega
$$

In this paper we explore three sentence embedding methods to implement $\xi$. We use a two-layer neural network as $M$ and employ the standard Mean Square Error (MSE) as the inverse $\psi^{-1}$, to determine the closest embedding of each class $\omega_{i} \in \Omega$ to the output of $M$.

\subsection{Adapting Kartsaklis Method (LSTM)}

Our basic inspiration for the algorithms of this paper is a text classification method proposed in (Kartsaklis et al., 2018) for the inverse dictionary problem where text definitions of terms are mapped to the term they define. The embedding of the class set into the continuous vector space (equivalent to the $\psi$ function in equation 3 ) is done by expanding the knowledge graph of the dictionary words with nodes corresponding to words related to those terms. Next, random walks are perfomed on the graph to compute graph embeddings related to each dictionary node, using the DeepWalk algorithm (Perozzi et al., 2014).

A Long Short-Term Memory (LSTM) neural network, composed of two layers and an attention mechanism, is used in (Kartsaklis et al., 2018) for mapping the input texts to the input embedding vector space. To map the two continuous vector spaces representing the definitions and the dictionary terms, a two-layer neural network $M$, learned from the training dataset, is used.

For this work, the knowledge graph is replaced by an intent proto-taxonomy $G$ which associates each class to a node and connects to them nodes which correspond to meta-knowledge concepts related to the class. To better capture the sequential 
aspect of the intent proto-taxonomies, we also connect each class node to bigrams of concepts, i.e., the concatenation of two subsequent concepts. We represent this by the function $\zeta$, such as $\zeta(\Omega)=G$, which is invertible. Substituting this in equation 3,

$$
S \stackrel{L S T M}{\rightarrow} \mathbb{R}^{n} \stackrel{M}{\rightarrow} \mathbb{R}^{m} \stackrel{\text { DeepWalk-1 }}{\rightarrow} G \stackrel{\zeta^{-1}}{\rightarrow} \Omega
$$

In practice, we compute the mapping from the class embedding space into the class set, called here $\operatorname{Inv} G: \mathbb{R}^{m} \rightarrow \Omega$, simply by determining the distance $d$ between the output point in $\mathbb{R}^{m}$ and the inverted projection of each class from $\Omega$ and then considering the closest class. That is, for each $w_{i} \in \Omega$, we consider the associated node in $G$ and compute the mapping in $\mathbb{R}^{m}$ of that node:

$$
\operatorname{Inv} G(x)=\underset{w_{i}}{\arg \min } d\left(x, \operatorname{DeepWalk}\left(G\left(w_{i}\right)\right)\right.
$$

By substituting this function into equation 4, we obtain the algorithm we call here $L S T M+T$ :

$$
S \stackrel{L S T M}{\rightarrow} \mathbb{R}^{n} \stackrel{M}{\rightarrow} \mathbb{R}^{m \stackrel{\operatorname{Inv} G}{\rightarrow}} \Omega
$$

For comparison, the traditional corresponding classification method is tested, where the graph embedding and associated functions are replaced by softmax+argmax. We call this LSTM:

$$
S \stackrel{L S T M}{\rightarrow} \mathbb{R}^{n} \stackrel{M}{\rightarrow} \mathbb{R}^{c \stackrel{\operatorname{softmax}}{\rightarrow}} \Omega
$$

\subsection{An Alternative to LSTM: USE}

Recently, several new general-purpose language models that can be used for computing sentence embeddings have been proposed, among them the Universal Sentence Enconder (USE) (Cer et al., 2018). Such an approach consists of a transformer neural network (Vaswani et al., 2017), trained on varied sources of data, such as Wikipedia, web news, web question-answer pages and discussion forum. USE has achieved state-of-the-art results in various tasks, so we decided to try it in our experiments as an alternative to the LSTM for the embedding of input sentences.

In this work we employed the version 3 of the multilingual USE ${ }^{2}$. By replacing LSTM with USE in eq. 6 we obtain algorithm $U S E+T$ :

$$
S \stackrel{U S E}{\rightarrow} \mathbb{R}^{n} \stackrel{M}{\rightarrow} \mathbb{R}^{m \stackrel{\operatorname{Inv} G}{\rightarrow}} \Omega
$$

\footnotetext{
${ }^{2}$ https://tfhub.dev/google/universal-sentence-encodermultilingual/3
}

Like in the previous case, we also compute the USE algorithm with traditional discrete softmax outputs for comparison, called here simply USE:

$$
S \stackrel{U S E}{\rightarrow} \mathbb{R}^{n} \stackrel{M}{\rightarrow} \mathbb{R}^{c} \stackrel{\text { softmax }}{\rightarrow} \Omega
$$

\subsection{Alternatives to DeepWalk}

To explore variants of algorithms for embedding the classes and also approaches which do not need to be trained from scratch and allow on-the-fly handling of meta-knowledge, we tried replacing DeepWalk with two different methods.

The first one consists of applying USE sentence embeddings also for the class embeddings, such as in eq. 10. To simplify notation, emb represents either LSTM or USE embeddings for the input text.

$$
S \stackrel{e m b}{\rightarrow} \mathbb{R}^{n} \stackrel{M}{\rightarrow} \mathbb{R}^{m \stackrel{U S E^{-1}}{\rightarrow}} G \stackrel{\zeta^{-1}}{\rightarrow} \Omega
$$

This approach is similar to the way DeepWalk works but instead of training the graph embeddings from scratch, the class embeddings are represented by the mean sentence embedding computed from different random walks starting in the class node. We name such methods $L S T M+S$ and $U S E+S$, for emb substituted by LSTM and USE, respectively.

Additionally, we also evaluate the replacement of DeepWalk by the Convolutional Deep Structured Semantic Model (CDSSM) proposed in (Chen et al., 2016), yielding the following algorithm where emb can be either LSTM or USE embeddings.

$$
S \stackrel{e m b}{\rightarrow} \mathbb{R}^{n} \stackrel{M}{\rightarrow} \mathbb{R}^{m \stackrel{C D S S M^{-1}}{\rightarrow}} G \stackrel{\zeta^{-1}}{\rightarrow} \Omega
$$

The CDSSM model consists of a three-layer convolutional neural network trained for creating embeddings of intent identifiers represented as sentences. In this work, we input to CDSSM the sequence of concepts listed in the nameId of each intent. We refer to these algorithms as $L S T M+C$ and $U S E+C$, for $e m b$ being substituted with LSTM and USE, respectively.

An intuitive way to understand those methods is to consider $U S E+T$ using a taxonomy as if its concepts had just abstract meanings: only their relations matter. In comparison, $U S E+S$ considers the meaning of the concepts besides their relations, while $U S E+C$ regards each nameId as a sentence, almost as if the developer had inputted a written description of the intent. 


\subsection{Out-of-Scope Sample Detection}

In this paper we are interested both in the problems of: (1) deciding whether an user utterance is inscope (IS) or out-of-scope (OOS) of the system; and (2) determining to which class an IS utterance belongs. For the former, a rejection mechanism based on a pre-defined threshold is used since it can be easily applied to all of the methods described previously without the need neither for any specific training procedure nor OOS training data.

In detail, suppose that for each class $\omega_{i} \in \Omega$ there is a score denoted $\phi_{i} \in Z$, where $|Z|=|\Omega|$. Given that $\max (Z)$ represents the highest score associated to a class and that a rejection threshold $\theta$ has been defined on a validation set, samples can be classified as OOS whenever $\max (Z)<\theta$. If so, they are simply rejected, i.e., no classification output is produced for them. Otherwise, the sample is considered as in-scope and the classification is conducted normally.

The scores in $Z$ are represented either by the softmax probability for the traditional softmax-based methods or by the similarity of sentence and intent embeddings for the proposed three approaches. For the latter, the similarity is computed by means of the dot product between the two embeddings.

\section{Metrics, Datasets, and Experiments}

In this section we present the experiments to evaluate the three algorithms described in the previous section, using each of the input embeddings LSTM and USE. We explore the impact on intent recognition both in terms of classifying correctly utterances (IS accuracy) and of finding which utterances are not covered by the intents (OOS accuracy).

\subsection{Evaluation metrics}

We employ a commonly-used metric for OOS dectection, equal error rate (EER) (Tan et al., 2019), which corresponds to the classification error rate when the threshold $\theta$ is set to a value where false acceptance rate (FAR) and false rejection rate (FRR) are the closest. These two metrics are defined as:

$$
\begin{gathered}
F A R=\frac{\text { number of accepted OOS samples }}{\text { total of OOS samples }} \\
F R R=\frac{\text { number of rejected IS samples }}{\text { total of IS samples }}
\end{gathered}
$$

In addition, in-scope error rate (ISER) is considered to report IS performance, i.e. the error rate considering only IS samples when $\theta$ is set to zero, similar to the class error rate in (Tan et al., 2019). This metric is important to evaluate whether the proposed classification methods are able to keep up with the performance of the baselines in the main classification task.

\subsection{The Larson and Telco Datasets}

During the development and initial testing of the algorithms, we used two English datasets for indepth experimentation. The first is the publiclyavailable Larson dataset (Larson et al., 2019); the second is a private real-world chatbot dataset used by a telecommunications provider for customer care, called here the Telco dataset. In the Larson dataset, we created an intent proto-taxonomy by hand, expanding the original identifiers of intents. The goal of the adjustments was to avoid spurious interference from taxonomy shortcomings or errors in the results. The complete list of the created taxonomic description of intents is listed in the appendix $\mathrm{C}$ to allow the reproduction of our results and further experimentation. In the Telco dataset, we created by hand the intent proto-taxonomy.

In the Larson dataset there is a total of 22,500 IS exemplars, evenly distributed across 150 classes, where 18,000 were used for training and 4,500 for testing. We conducted a simulation of OOS detection with the IS exemplars by doing 5 random samplings where we took out 30 intents and 3,600 training exemplars. We trained only with the remaining 120 intents and 14,400 exemplars. The test was then conducted using all the non-used 4,500 exemplars, where the 3,600 associated to the trained classes were considered the IS samples and the remaining 900 became OOS samples.

The Telco dataset contains 4,093 exemplars and 87 intents. From those, 3,069 exemplars were used for training and 1,024 for testing. The OOS scenario was simulated by extracting different random samplings where 5 intents were removed. Given the smaller size of this dataset compared to Larson, we conducted 20 samplings instead of 5 .

For both sets we considered the following setup defined after preliminary evaluations. For the LSTM-based methods, the input sentence embedding size was set to 150 and output embeddings to 200. DeepWalk walk sizes were set to 20 for LSTM+T and USE+T. For both USE- and softmaxbased methods we trained a two-layer neural network with 800 hidden neurons for 50 epochs. 


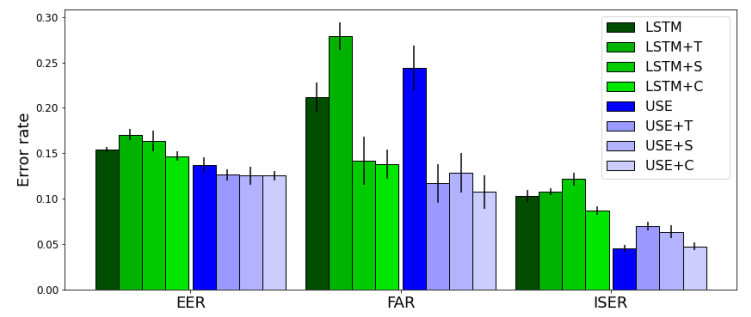

Figure 3: Different methods to incorporate the intent proto-taxonomy in Larson dataset, compared to the LSTM and USE baselines.

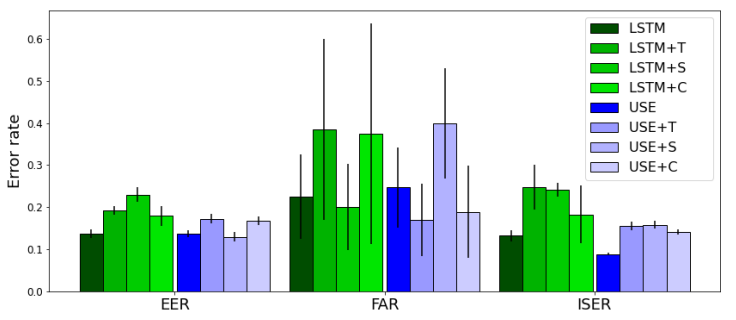

Figure 4: Different methods to incorporate the intent proto-taxonomy in Telco dataset, compared to the LSTM and USE baselines.

\subsection{Results in the Larson and Telco Datasets}

The results on the Larson dataset are graphically depicted in fig. 3. We observed that there was a slight improvement (a decrease) in EER, especially with the USE-based and the LSTM+C methods. More notably, there was a significant improvement in terms of FAR for all USE-based methods and $\mathrm{LSTM}+\mathrm{S}$ and LSTM+C. Notice that even though the proposed approaches generally did not outperform LSTM and USE in ISER (except LSTM+C), we observed that the methods with better ISER tended to produce also better EER and FAR.

In fig. 4, we see that the results on the Telco dataset presented a different scenario. The proposed methods generally performed worse than or, at best, similar to LSTM and USE in EER. In terms of FAR, some methods such as USE+T and USE+C seem to outperform the others but, considering the high standard deviations, the results were not significant. On the other hand, we also observed that the methods failed to get close in ISER compared to the softmax-based methods. That seems to indicate that for the cases where making use of metaknowledge harms too much ISER, the symbolic knowledge did not decrease neither EER nor FAR.

There were two key findings from our experiments with the Larson and the Telco datasets. First, the improvements using LSTM or USE as a base- line seemed to be similar, possibly slightly better for the USE algorithm. Second, and most importantly, we saw much more improvement in the use of the intent proto-taxonomy in the Larson than in the Telco dataset, in spite of the similar nature of the datasets and the intent proto-taxonomies. This motivated us to try out the ideas in a larger and more diverse number of workspaces and solely focusing on USE to simplify the experiments.

\subsection{The ChatWorks Dataset}

To test our algorithms in a context of high diversity and realism, we used the same large set of real, professional workspaces explored in (Pinhanez et al., 2021), which come from the professional chatbot development platform ChatWorks.

We started with the 3,840 workspaces available in English. To eliminate possible problems due to workspaces with poor quality, we employed the $3 \sigma$-rule, where values smaller greater than 3 standard deviations from the mean are not considered.Workspaces with the number of intents or exemplars below and above those thresholds were removed. Also, to avoid workspaces with few exemplars per intent, the ratio of the number of exemplars to the number of intents had to be greater than 10. From the filtered set we randomly selected 200 workspaces for testing.

The evaluation involved the execution of 20 iterations for each workspace. The tests were performed for all USE-based methods (USE, USE+T, USE+S, and USE+C). First, the workspaces were split into training and test datasets (75\% and $25 \%$, respectively). Next, the four methods were trained and tested on these datasets. The evaluation metrics (EER, FAR, and ISER) were then measured on the results for the test datasets and the average errors and their standard deviations were computed.

\subsection{Results in the ChatWorks Dataset}

Appendix D contains a table with the results for each of the 200 workspaces in the ChatWorks dataset. Figure 5 summarizes the results of the experiments showing the distribution of the 200 workspaces according to ranges of the improvement of each of the three methods compared to the baseline of USE. Improvement is calculated by subtracting the errors in each of our proposed methods from the errors in the USE baseline (error values are scores between 0 and 1). When one of our methods was worse than the baseline then diff $<0$, 
since smaller is better, and conversely for when it is better than the baseline, i.e., diff $\geqslant 0$.

The results shown in fig. 5 indicate that the USE+C algorithm achieved the best results in all three metrics, although there is a significant portion of workspaces where the other methods also did well, especially in OOS detection (FAR).

But, more important, the results seem to support our claim that meta-knowledge embedded in the output layer of our neuro-symbolic algorithms can improve intent recognition performance in practical systems. Notably in OOS detection (FAR), 67\% of the workspaces experienced a decrease in the error rate using USE+C. Besides, in 39\% of the workspaces we observed a decrease in the error rate of more than 0.05 (in a 0 to 1 scale), and in $23 \%$, of more than 0.1 . The USE+T also did well with similar but slightly smaller decreases in error.

Overall, the error rates for the EER metric also decreased in relation to the baseline. Figure 5 shows that $41 \%$ of the workspaces had some level of decrease in EER with the USE+C algorithm, in $10 \%$ of them with decreases of 0.05 or more. However, the results for the in-scope accuracy (ISER) were much smaller with only about $16 \%$ of the workspaces having any kind of decrease.

The ChatWorks dataset, as noted before, includes all kinds of workspaces. Taxonomy rates varies anywhere from 0 to 1 , and there are very small and very large workspaces. To test our methods in a scenario closer to a professional, welldeveloped chatbot, we filtered further the dataset to include only workspaces with taxonomy rate greater or equal to 0.7 , with number of intents equal or more than 32, and at least an average of 25 exemplars per intent, resulting in 18 workspaces.

Figure 6 shows the distribution of the results of the experiments with those 18 workspaces, which were better than in the full ChatWorks dataset. Both USE+T and USE+C yielded EER decreases in 50\% or more of the workspaces. Moreover, $83 \%$ of the workspaces decreased the FAR error, either with USE+T or USE+C, and both decreased FAR in more than a third of the workspaces by more than 0.1 . We discuss the results and implications next.

\section{Discussion and Future Work}

We started this paper by proposing the combination of exemplars and symbolic characterizations of a class as a way to enhance ML-based intent recognition. We proposed 3 new neuro-symbolic algo-

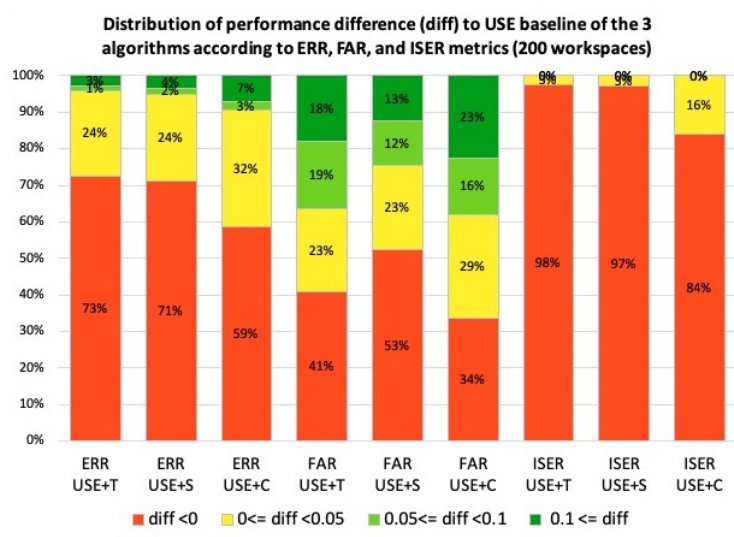

Figure 5: Distribution of performance difference (diff) to USE baseline of the 3 methods according to EER, FAR, and ISER metrics in all 200 workspaces of the Chatworks dataset.

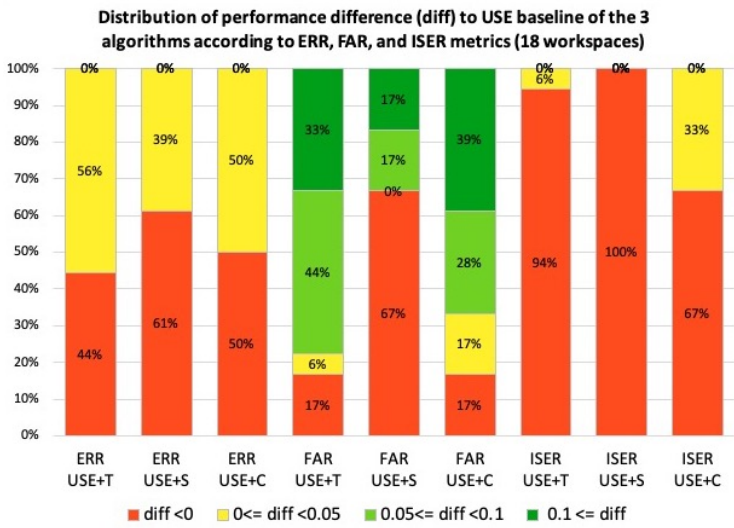

Figure 6: Distribution of performance difference (diff) to USE baseline of the 3 methods according to EER, FAR, and ISER metrics in the 18 most developed workspaces of the Chatworks dataset.

rithms and tested them using datasets built using data from intent identifiers of conversational systems. Such identifiers often store taxonomic-like structures, due to a common practice among developers of professional conversational systems (Pinhanez et al., 2021). The results of the experiments indicate that the intent proto-taxonomies embedded by those developers can indeed be used by many workspaces to improve accuracy in intent recognition, notably in OOS detection.

We see as one of the main contributions of this paper the creation of methods with which ML engineers can improve the accuracy of their systems by simply mining "documentation" from chatbots, without any further data and annotation.

Our results show that almost $40 \%$ of the 200 professional workspaces drawn from ChatWorks saw decreases of more than 0.05 in OOS detection 
error rates. Also, in $42 \%$ of them the overall error rate was improved, using the USE+C algorithm. When considering the more well-structured and developed 18 workspaces, we saw much higher gains with the USE+T algorithm. Those accuracy improvements were achieved without any change in the training set but simply by incorporating the meta-knowledge into intent recognition.

Notice that the testing methodology used in this work is considerably harder than the practice of the majority of research papers, since it evaluates performance in 200 professional, non-edited workspaces from different domains. In reality, most ML algorithms do not perform well in all datasets, and ML practitioners often test different algorithms and parameters until accuracy is good enough.

However, the improvement in OOS detection (FAR) was not mirrored in classification error (ISER). First, we must keep in mind that intent classification is often performed in two steps, first OOS sentences detection and removal, followed by intent classification of the IS sentences. Given the improvements observed in OOS detection, it would make sense to use our algorithms in the first step for many of the ChatWorks workspaces (about 60\% of them), and selectively use it for IS classification only when it works better than the baseline.

But why were there so many workspaces where we did not see impact? It is important to take into account that the ChatWorks dataset has workspaces in different stages of development and deployment. By selecting better quality workspaces, we saw much higher gains. We explored briefly characterizations of the intent proto-taxonomy quality, such as taxonomy rate, depth of the taxonomy, and number of concepts, but we saw no clear correlation with decreases in error rates. We believe more complex metrics of knowledge structure need to be employed to characterize which intent prototaxonomies are likely to have the greatest impacts. We plan to do so in our future work.

It is important to notice that, in the workspaces where we did see impact, the symbolic knowledge was mined from an absolutely "raw" format. In spite of that, by using the basic graph mining method described in appendix A, it was possible to obtain a "meaningful" taxonomic structure, similar to a knowledge graph which could be used by our neuro-symbolic algorithms. To improve the quality of the taxonomies, we are working on designing an interface which allows the developers to manipu- late directly the intent proto-taxonomy to make it more correct and complete, so to possibly decrease even more the intent recognition error rates.

We have demonstrated in this work that combining exemplar and symbolic ways of defining classes can have a positive impact in the performance of the recognition system. This was done in the context of conversational systems where developers fortuitously embed such alternative descriptions of classes in their name identifiers. We believe it is possible to find in other machine learning development platforms similar patterns of knowledge embedding.

For example, we know that it is common for people to use similar taxonomic structures when naming file and e-mail folders, giving names to functions and variables in programs and data, and writing comments into Jupyter notebooks. Also, ML platforms can further foster the use of metadata by developers by explicitly asking them to input, besides exemplars, categorical or textual descriptions of the classes.

As we move along the path of creating such neuro-symbolic systems, not only we should expect that the job of developers becomes easier, as they follow their own cultural and linguistic practices, but also that machines became better in recognizing those classes accurately. Using multiple forms of class definitions can be a winning proposition for both ML systems and their developers.

\section{Ethical Issues}

The ChatWorks dataset was composed only of workspaces in which the developers explicitly opted-in to share their code and content for research and development purposes with the company which owns the platform. Those workspaces were shared by the company with the authors of this paper with a clear condition of not publicly sharing their contents and publishing only aggregated results or in an anonymous form. We do not see any specific impact of those limitations in the results of our research but they preclude easy forms of replication of our results with that dataset. To better enable reproducibility, we presented the analysis of the public Larson dataset and shared the intent prototaxonomy we created manually from its original intent structure in appendix $\mathrm{C}$. 


\section{References}

Zeynep Akata, Florent Perronnin, Zaid Harchaoui, and Cordelia Schmid. 2016. Label-embedding for image classification. IEEE Transactions on Pattern Analysis and Machine Intelligence, 38(7):1425-1438.

Zeynep Akata, Scott Reed, Daniel Walter, Honglak Lee, and Bernt Schiele. 2015. Evaluation of output embeddings for fine-grained image classification. In Proceedings of the IEEE Conference on Computer Vision and Pattern Recognition (CVPR).

Yoshua Bengio. 2017. The consciousness prior.

Tarek R. Besold, Artur d'Avila Garcez, Sebastian Bader, Howard Bowman, Pedro Domingos, Pascal Hitzler, Kai-Uwe Kuehnberger, Luis C. Lamb, Daniel Lowd, Priscila Machado Vieira Lima, Leo de Penning, Gadi Pinkas, Hoifung Poon, and Gerson Zaverucha. 2017. Neural-symbolic learning and reasoning: A survey and interpretation.

Geoffrey C Bowker and Susan Leigh Star. 2000. Sorting things out: Classification and its consequences. MIT press.

Paulo Cavalin, Victor Henrique Alves Ribeiro, Ana Appel, and Claudio Pinhanez. 2020. Improving out-ofscope detection in intent classification by using embeddings of the word graph space of the classes. In Proceedings of the 2020 Conference on Empirical Methods in Natural Language Processing (EMNLP), pages 3952-3961, Online. Association for Computational Linguistics.

Daniel Cer, Yinfei Yang, Sheng-yi Kong, Nan Hua, Nicole Limtiaco, Rhomni St. John, Noah Constant, Mario Guajardo-Cespedes, Steve Yuan, Chris Tar, Brian Strope, and Ray Kurzweil. 2018. Universal sentence encoder for English. In Proceedings of the 2018 Conference on Empirical Methods in Natural Language Processing: System Demonstrations, pages 169-174, Brussels, Belgium. Association for Computational Linguistics.

Y. Chen, D. Hakkani-Tür, and X. He. 2016. Zeroshot learning of intent embeddings for expansion by convolutional deep structured semantic models. In 2016 IEEE International Conference on Acoustics, Speech and Signal Processing (ICASSP), pages 6045-6049.

Andrea Civan, William Jones, Predrag Klasnja, and Harry Bruce. 2008. Better to organize personal information by folders or by tags?: The devil is in the details. Proceedings of the American Society for Information Science and Technology, 45(1):1-13.

Luc De Raedt, R. Manhaeve, S. Dumancic, Thomas Demeester, and A. Kimmig. 2019. Neuro-symbolic = neural + logical + probabilistic. In Proceedings of the 2019 International Workshop on Neural- Symbolic Learning and Reasoning, page 4.
Emile Durkheim and Marcel Mauss. 1963. Primitive Classification, volume 273. University of Chicago Press.

Lisa Ehrlinger and Wolfram Wöß. 2016. Towards a definition of knowledge graphs. SEMANTiCS (Posters, Demos, SuCCESS), 48:1-4.

Marco Fossati, Dimitris Kontokostas, and Jens Lehmann. 2015. Unsupervised learning of an extensive and usable taxonomy for dbpedia. In Proceedings of the 11th International Conference on Semantic Systems, SEM '15. ACM.

Artur d'Avila Garcez, Marco Gori, Luis C. Lamb, Luciano Serafini, Michael Spranger, and Son N. Tran. 2019. Neural-symbolic computing: An effective methodology for principled integration of machine learning and reasoning. Journal of Applied Logics, 6(4):611-631.

Drew Hudson and Christopher D Manning. 2019a. Learning by abstraction: The neural state machine. In Advances in Neural Information Processing Systems, pages 5903-5916.

Drew Hudson and Christopher D Manning. 2019b. Learning by abstraction: The neural state machine. In H. Wallach, H. Larochelle, A. Beygelzimer, F. d'Alché-Buc, E. Fox, and R. Garnett, editors, $A d$ vances in Neural Information Processing Systems 32, pages 5903-5916. Curran Associates, Inc.

Shaoxiong Ji, Shirui Pan, Erik Cambria, Pekka Marttinen, and Philip S. Yu. 2020. A survey on knowledge graphs: Representation, acquisition and applications.

Daniel Jurafsky and James H. Martin. 2009. Speech and Language Processing (2nd Edition). PrenticeHall, Inc., USA.

Dimitri Kartsaklis, Mohammad Taher Pilehvar, and Nigel Collier. 2018. Mapping text to knowledge graph entities using multi-sense LSTMs. In Proceedings of the 2018 Conference on Empirical Methods in Natural Language Processing, pages 19591970, Brussels, Belgium. Association for Computational Linguistics.

Stephen M Kosslyn. 2006. You can play 20 questions with nature and win: Categorical versus coordinate spatial relations as a case study. Neuropsychologia, 44(9):1519-1523.

Stefan Larson, Anish Mahendran, Joseph J. Peper, Christopher Clarke, Andrew Lee, Parker Hill, Jonathan K. Kummerfeld, Kevin Leach, Michael A. Laurenzano, Lingjia Tang, and Jason Mars. 2019. An evaluation dataset for intent classification and out-of-scope prediction. In Proceedings of the 2019 Conference on Empirical Methods in Natural Language Processing and the 9th International Joint Conference on Natural Language Processing (EMNLP-IJCNLP), pages 1311-1316, Hong Kong, China. Association for Computational Linguistics. 
Robin Manhaeve, Sebastijan Dumancic, Angelika Kimmig, Thomas Demeester, and Luc De Raedt. 2018. Deepproblog: Neural probabilistic logic programming. In S. Bengio, H. Wallach, H. Larochelle, K. Grauman, N. Cesa-Bianchi, and R. Garnett, editors, Advances in Neural Information Processing Systems 31, pages 3749-3759. Curran Associates, Inc.

Christopher Manning and Hinrich Schutze. 1999. Foundations of statistical natural language processing. MIT press.

Jiayuan Mao, Chuang Gan, Pushmeet Kohli, Joshua B. Tenenbaum, and Jiajun Wu. 2019. The NeuroSymbolic Concept Learner: Interpreting Scenes, Words, and Sentences From Natural Supervision. In International Conference on Learning Representations.

Rodney Needham. 1979. Symbolic classification. Goodyear Publishing Company.

Allen Newell. 1973. You can't play 20 questions with nature and win: Projective comments on the papers of this symposium. In W.G. Chase, editor, Visual information processing. Academic Press.

Alessandro Oltramari, Jonathan Francis, Cory Henson, Kaixin Ma, and Ruwan Wickramarachchi. 2020. Neuro-symbolic architectures for context understanding.

Mark Palatucci, Dean Pomerleau, Geoffrey E Hinton, and Tom M Mitchell. 2009. Zero-shot learning with semantic output codes. In Advances in Neural Information Processing Systems, volume 22. Curran Associates, Inc.

Emilio Parisotto, Abdel-Rahman Mohamed, Rishabh Singh, Lihong Li, Dengyong Zhou, and Pushmeet Kohli. 2017. Neuro-symbolic program synthesis. In International Conference on Learning Representations (ICLR).

Bryan Perozzi, Rami Al-Rfou, and Steven Skiena. 2014. Deepwalk: Online learning of social representations. In Proceedings of the 20th ACM SIGKDD international conference on Knowledge discovery and data mining, pages 701-710.

Claudio Santos Pinhanez, Heloisa Candello, Paulo Cavalin, Mauro Carlos Pichiliani, Ana Paula Appel, Victor Henrique Alves Ribeiro, Julio Nogima, Maira de Bayser, Melina Guerra, Henrique Ferreira, et al. 2021. Integrating machine learning data with symbolic knowledge from collaboration practices of curators to improve conversational systems. In Proceedings of the 2021 ACM Conference on Human Factors in Computing Systems (CHI'21), pages 113.

Whitman Richards. 1982. How to play twenty questions with nature and win.
Richard Socher, Milind Ganjoo, Christopher D Manning, and Andrew Ng. 2013. Zero-shot learning through cross-modal transfer. In Advances in Neural Information Processing Systems, volume 26. Curran Associates, Inc.

Ming Tan, Yang Yu, Haoyu Wang, Dakuo Wang, Saloni Potdar, Shiyu Chang, and Mo Yu. 2019. Out-ofdomain detection for low-resource text classification tasks. In Proceedings of the 2019 Conference on Empirical Methods in Natural Language Processing and the 9th International Joint Conference on Natural Language Processing (EMNLP-IJCNLP), pages 3566-3572, Hong Kong, China. Association for Computational Linguistics.

Joshua B. Tenenbaum, Charles Kemp, Thomas L. Griffiths, and Noah D. Goodman. 2011. How to grow a mind: Statistics, structure, and abstraction. Science, 331(6022):1279-1285.

Ashish Vaswani, Noam Shazeer, Niki Parmar, Jakob Uszkoreit, Llion Jones, Aidan N Gomez, Łukasz Kaiser, and Illia Polosukhin. 2017. Attention is all you need. In Advances in neural information processing systems, pages 5998-6008.

Wei Wang, Vincent W Zheng, Han Yu, and Chunyan Miao. 2019. A survey of zero-shot learning: Settings, methods, and applications. ACM Transactions on Intelligent Systems and Technology (TIST), 10(2):1-37

Steve Whittaker, Tara Matthews, Julian Cerruti, Hernan Badenes, and John Tang. 2011. Am I wasting my time organizing email? a study of email refinding. In Proceedings of the SIGCHI Conference on $\mathrm{Hu}$ man Factors in Computing Systems, CHI '11, page 3449-3458, New York, NY, USA. Association for Computing Machinery.

Bai Yang, Zhang Liping, and Zhao Fengrong. 2019. A survey on research of code comment. In Proceedings of the 2019 3rd International Conference on Management Engineering, Software Engineering and Service Sciences, ICMSS 2019, page 45-51, New York, NY, USA. Association for Computing Machinery. 


\section{A An Algorithm to Extract Intent Proto-Taxonomies from NameIds}

In our previous work (Pinhanez et al., 2021) we described an algorithm to mine proto-taxonomies from the nameIds of a workspace. For completeness, we include it here. It consists of three steps:

1. Finding the best separator to split the name into a sequence of concepts;

2. Splitting the nameIds with the selected separator.

3. Generating an intent proto-taxonomy using the terms split by the best separator as concepts and considering consecutive concepts in a nameId as having a link between them.

In order to find the best separator, our algorithm first calculates the perplexity of the bag of concepts using each separator. Perplexity is a measure of uncertainty for a given sequence of words (or concepts) appearing in a language model (Manning and Schutze, 1999). For that, we build probabilistic language models based on bigrams using the maximum likelihood estimator as in (Jurafsky and Martin, 2009, Chapter 3), and then compute the average perplexity using a standard leave-oneintent-out evaluation scheme. The separator which minimizes perplexity is chosen as the separator for the workspace.

\section{B Intent Proto-Taxonomies as a Common Practice in ChatWorks}

In (Pinhanez et al., 2021) we evaluated the use of proto-taxonomies by the curators of the ChatWorks dataset, by developing a metric, called taxonomy rate, which is the ratio of the number of nameIds which have a proto-taxonomy embedded in it to the total number of intents of the workspace.

To determine whether a given nameId has a proto-taxonomy embedded in it, we considered three criteria: (1) the nameId has two or more concepts; (2) there is at least one other nameId which has at least one identical concept at the exact same level; and (3) the concept does not appear in all nameIds of the workspace in that level. After all the nameIds of a workspace were determined as having an embedded intent or not, the taxonomy rate was calculated by considering the ratio between the number of intents with a taxonomic structure and the number of intents of the workspace.

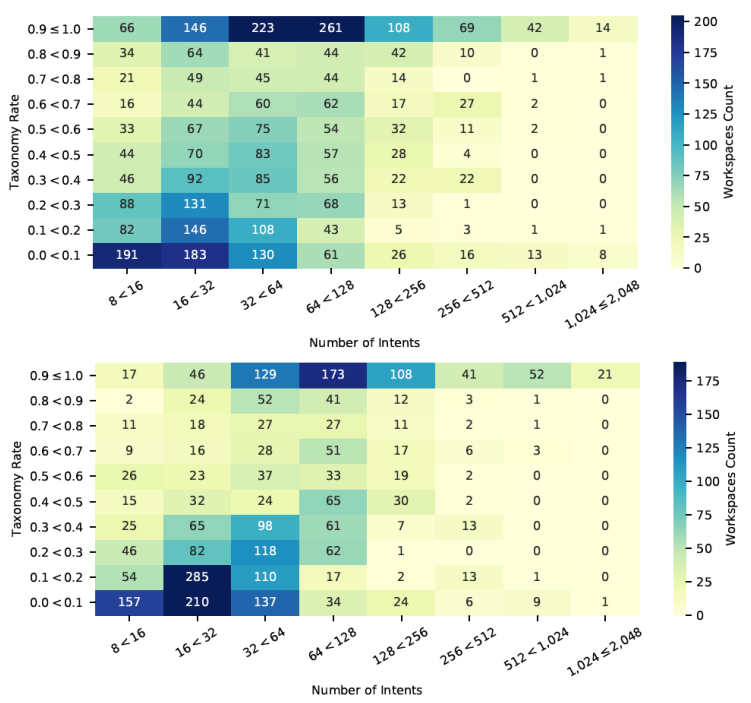

Figure 7: Distribution of the number of workspaces according to the taxonomy rate of the 3,840 English (top) and 2,895 Portuguese (bottom) workspaces. Notice that the $\mathrm{X}$ axis is in logarithmic scale.

A taxonomy rate of above $10 \%$ was observed in $76 \%$ of the English workspaces and in $80 \%$ the Portuguese ones. Almost 52\% of all workspaces, in English, and 38\% in Portuguese, had a taxonomy rate larger than $50 \%$. Moreover, $16 \%$ and $20 \%$ of the workspaces, for English and Portuguese respectively, had a very high taxonomy rate from $90 \%$ to $100 \%$. Considering both the English and Portuguese workspaces, about $70 \%$ of them have a taxonomy rate above $20 \%$ and more than half of the workspaces above 64 intents have a taxonomy of $50 \%$ or more.

Figure 7 shows how the workspaces are distributed considering both the number of intents ( $\mathrm{X}$ axis) and the taxonomy rate ( $\mathrm{Y}$ axis). Notice that the distribution, in both languages, follows a sort of a "step" function where, as the threshold between 32 and 64 intents is crossed, the majority of the workspaces has more than 50\% of taxonomy rate. More details can be found in (Pinhanez et al., 2021).

\section{The Intent Proto-Taxonomy Created for the Larson Dataset}

In tables 1 and 2, we list the taxonomy which was manually created for the Larson dataset, with the original nameId on the left and the created taxonomic representation on the right, represented as a string of concepts separated by spaces. 


\begin{tabular}{|c|c|}
\hline nameId & $\begin{array}{l}\text { Concepts } \\
\end{array}$ \\
\hline accept_reservations & accept reservation \\
\hline account_blocked & account blocked \\
\hline alarm & set alarm \\
\hline application_status & application status \\
\hline apr & what month \\
\hline are_you_a_bot & you bot \\
\hline balance & what balance \\
\hline bill_balance & what bill balance \\
\hline bill_due & when bill due \\
\hline book_flight & book flight \\
\hline book_hotel & book hotel \\
\hline calculator & calculate \\
\hline calendar & calendar \\
\hline calendar_update & calendar update \\
\hline calories & calories dish \\
\hline cancel & cancel action \\
\hline cancel_reservation & cancel reservation \\
\hline car_rental & car rental \\
\hline card_declined & card declined \\
\hline carry_on & carry-on rule \\
\hline change_accent & change accent \\
\hline change_ai_name & change bot name \\
\hline change_language & change language \\
\hline change_speed & change speed \\
\hline change_user_name & change user name \\
\hline change_volume & change volume \\
\hline confirm_reservation & confirm reservation \\
\hline cook_time & cook time \\
\hline credit_limit & credit limit \\
\hline credit_limit_change & credit limit change \\
\hline credit_score & credit score \\
\hline current_location & what current location \\
\hline damaged_card & damaged card \\
\hline date & what date \\
\hline definition & definition \\
\hline direct_deposit & direct deposit \\
\hline directions & what direction \\
\hline distance & what distance \\
\hline do_you_have_pets & do you have pet \\
\hline exchange_rate & exchange rate \\
\hline expiration_date & expiration date \\
\hline find_phone & find phone \\
\hline flight_status & flight status \\
\hline flip_coin & flip coin \\
\hline food_last & food last \\
\hline freeze_account & block account \\
\hline fun_fact & fun fact \\
\hline gas & gas level \\
\hline gas_type & gas type \\
\hline goodbye & goodbye \\
\hline greeting & greeting \\
\hline how_busy & how busy \\
\hline how_old_are_you & how old you \\
\hline improve_credit_score & improve credit score \\
\hline income & what income \\
\hline ingredient_substitution & ingredient substitution \\
\hline ingredients_list & ingredient list \\
\hline insurance & insurance benefit \\
\hline insurance_change & insurance change \\
\hline interest_rate & interest rate \\
\hline international_fees & international fee \\
\hline international_visa & international visa \\
\hline jump_start & jump start \\
\hline last_maintenance & last maintenance \\
\hline lost_luggage & lost luggage \\
\hline make_call & make call \\
\hline maybe & maybe \\
\hline meal_suggestion & meal suggestion \\
\hline meaning_of_life & what meaning life \\
\hline measurement_conversion & measurement conversion \\
\hline meeting_schedule & meeting schedule \\
\hline min_payment & minimum payment \\
\hline $\mathrm{mpg}$ & what mpg \\
\hline new_card & apply card \\
\hline next_holiday & next holiday \\
\hline next_song & next song \\
\hline & no \\
\hline nutrition_info & nutrition info \\
\hline oil_change_how & how change oil \\
\hline oil_change_when & when change oil \\
\hline order & order shopping \\
\hline order_checks & order check \\
\hline order_status & order status \\
\hline pay_bill & pay bill \\
\hline payday & when payday \\
\hline pin_change & change pin \\
\hline play_music & play music \\
\hline plug_type & what plug type \\
\hline pto_balance & pto balance \\
\hline pto_request & pto request \\
\hline & $\ldots$ \\
\hline
\end{tabular}

Table 1: The taxonomy created for the Larson dataset.

\begin{tabular}{|ll|}
\hline nameId & Concepts \\
\hline pto_request_status & pto request status \\
pto_used & pto used \\
recipe & recipe dish \\
redeem_rewards & redeem reward \\
reminder & reminder action \\
reminder_update & reminder update \\
repeat & repeat action \\
replacement_card_duration & replacement card duration \\
report_fraud & report fraud \\
report_lost_card & report lost card \\
reset_settings & reset setting \\
restaurant_reservation & restaurant reservation \\
restaurant_reviews & restaurant review \\
restaurant_suggestion & restaurant suggestion \\
rewards_balance & reward balance \\
roll_dice & roll dice \\
rollover_401k & rollover 401k \\
routing & find routing \\
schedule_maintenance & schedule maintenance \\
schedule_meeting & schedule meeting \\
share_location & share location \\
shopping_list & shopping list \\
shopping_list_update & shopping list update \\
smart_home & smart home \\
spelling & spelling word \\
spending_history & spending history \\
sync_device & sync device \\
taxes & what taxes \\
tell_joke & tell joke \\
text & text person \\
thank_you & thank \\
time & what time \\
timer & set timer \\
timezone & set timezone \\
tire_change & tire change \\
tire_pressure & tire pressure \\
todo_list & todo list \\
todo_list_update & todo list update \\
traffic & what traffic \\
transactions & card transaction \\
transfer & transfer account \\
translate & translate word \\
travel_alert & travel alert \\
travel_notification & travel notification \\
travel_suggestion & travel suggestion \\
uber & get uber \\
update_playlist & update playlist \\
user_name & user name \\
vaccines & ghat vaccine \\
w2 & what weather \\
weather & what hobby \\
what_are_your_hobbies & what ask you \\
what_can_i_ask_you & what name \\
what_is_your_name & what song \\
what_song & where you from \\
where_are_you_from & whisper mode \\
whisper_mode & who you work \\
who_do_you_work_for & who made you \\
who_made_you & . \\
yes & \\
\hline
\end{tabular}

Table 2: The taxonomy created for the Larson dataset (cont.).

\section{Results of the Proposed Algorithms in the ChatWorks Dataset}

Table 3 shows the results of the proposed three algorithms and the baseline for each workspace in the ChatWorks dataset. For each metrics, EER, FAR, and ISER, the table shows the average accuracy and the standard deviation over the experiments made with the 20 random splits. The table also lists information characterizing the workspaces: number of intents, number of exemplars, the average number of exemplars per intent, taxonomy rate, the average depth of the taxonomy graph, and the number of different concepts. 


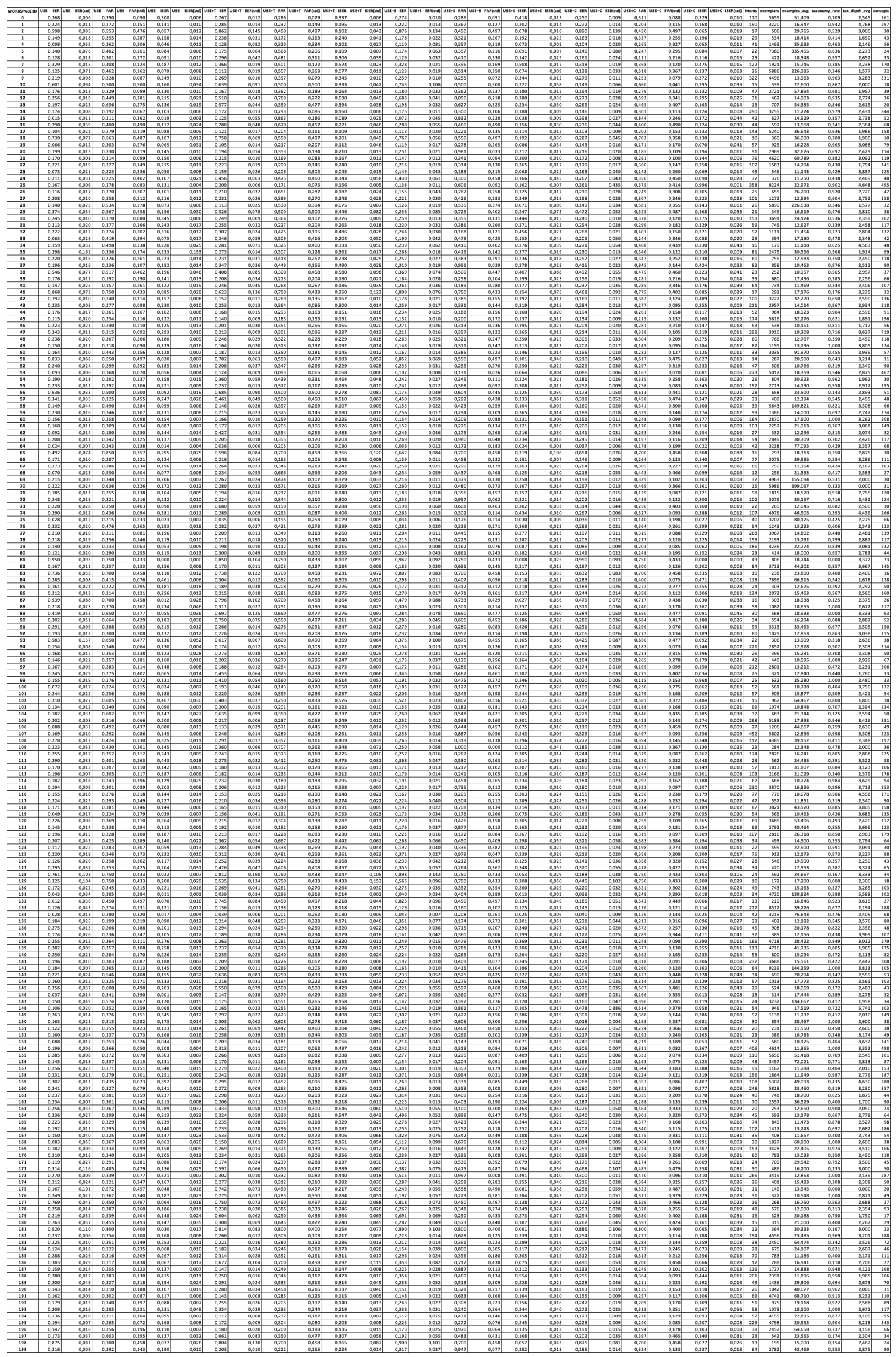

Table 3: The results of the 4 algorithms for each workspace in the ChatWorks dataset, including the average accuracy and the standard deviation ( 20 random splits). The table also lists information characterizing each workspace. 